\title{
Rencontre avec l'histoire des femmes et du féminisme : itinéraires de Japonaises francophiles
}

Groupe Histoire des femmes

\section{(2) OpenEdition}

1 Journals

Édition électronique

URL : https://journals.openedition.org/clio/4742

DOI : $10.4000 /$ clio.4742

ISSN : 1777-5299

Éditeur

Belin

Édition imprimée

Date de publication : 1 novembre 2006

ISBN : 2-85816-867-9

ISSN : $1252-7017$

Référence électronique

Groupe Histoire des femmes, «Rencontre avec l'histoire des femmes et du féminisme : itinéraires de Japonaises francophiles », Clio. Histoire, femmes et sociétés [En ligne], 24 | 2006, mis en ligne le 23 août 2013, consulté le 22 avril 2022. URL : http://journals.openedition.org/clio/4742 ; DOI : https://doi.org/ 10.4000/clio.4742

Ce document a été généré automatiquement le 22 avril 2022

Tous droits réservés 


\title{
Rencontre avec l'histoire des femmes et du féminisme : itinéraires de Japonaises francophiles
}

\author{
Groupe Histoire des femmes
}

1 L'histoire des femmes, née de la brèche ouverte par les mouvements féministes de la fin $\mathrm{du} \mathrm{XX}^{\mathrm{e}}$ siècle, occupe un champ de moins en moins contesté dans la recherche historique. Pourtant cette histoire reste généralement peu ou mal enseignée. Mais si l'histoire des femmes intéresse, que savons-nous des réactions des lectrices à cette histoire, de quelle manière ces lectures ont-elles pu influencer leurs façons de vivre ? À partir de l'exemple d'un espace de rencontre et d'échange entre Françaises et Japonaises, créé en 1983, nous voudrions montrer comment la réflexion sur l'histoire et sur la place que les femmes y occupent a pu jouer un rôle libérateur. Au départ, un groupe de Japonaises captivées par la culture et l'histoire françaises, à l'arrivée, une prise de conscience féministe. L'élan de curiosité soulevé par une culture étrangère, se muant au fil des recherches communes en réappropriation de l'histoire des femmes, a abouti à éclairer d'un jour nouveau les itinéraires de chacune. Le travail sans cesse approfondi visant à replacer ses expériences personnelles dans un vécu collectif a suscité une nouvelle prise de conscience; les unes et les autres ont compris qu'elles avaient été, chacune à sa place, actrices de cette histoire des femmes, que leur vie en avait constitué la trame et qu'en conséquence, elles pouvaient encore agir sur son cours.

2 Durant vingt années de rencontres informelles, les participantes du groupe ont réussi à créer un nouvel espace de réflexion à la croisée des intérêts des chercheuses et du public féminin attiré par l'histoire. Aussi, ont-elles désiré faire part des résultats de leur travail. En retraçant l'histoire d'un groupe de femmes, de son fonctionnement et de son évolution féministe, cet article collectif, destiné plus particulièrement à un 
lectorat français, veut montrer la possibilité d'un usage vivant et dynamique de l'histoire des femmes. ${ }^{1}$

\section{La Société Franco-japonaise des Études sur les Femmes (SFJEF)}

Pour comprendre l'originalité de cette société franco-japonaise ${ }^{2}$ qui depuis plus de vingt ans s'est donnée pour objectif de développer nos connaissances sur les femmes des deux pays, il faut remonter brièvement aux années 1950, au cours desquelles le Japon se reconstruit à la suite de la défaite. En 1945, les Japonaises obtiennent le droit de vote, mais leurs préoccupations majeures concernent alors la survie et la vie quotidienne. Ce n'est qu'à partir des années 1960 que les féministes commencent à dénoncer les discriminations sexuelles les plus évidentes. Les militantes, influencées par le réveil du féminisme international, contestent la place réservée aux femmes. Né dans le sillage des mouvements d'étudiants de 1968, le Women's Lib japonais se montre très radical, mais moins efficace que le courant majoritaire plus modéré.

Les fondatrices de la Société franco-japonaise appartiennent à ce courant qui opte pour l'étude aux dépens de l'action directe. Celles qui avaient fait des études de français étaient curieuses de connaitre la situation des femmes en France et en Europe, largement ignorée jusque-là. La documentation faisait défaut. Pour combler cette lacune, et grâce à la possibilité croissante de voyager entre les deux pays dans les années 1980, un petit groupe de Japonaises, éditrices, professeurs, personnalités de gauche créèrent un fond de documentation ouvert à toutes les femmes intéressées par la France indépendamment de leur qualification professionnelle ou de leur appartenance idéologique. Cette initiative fut un succès. En 1994, le groupe prit le nom de Société Franco-japonaise des Études sur les Femmes. Aujourd'hui, avec 170 membres français et japonais ainsi qu'une revue annuelle Espace des Femmes, la Société fait partie de la Maison Franco-japonaise de Tokyo, ce qui lui assure une dimension académique reconnue. Son institutionnalisation n'a pas tué l'originalité de la SFJEF, elle demeure un centre de recherches toujours ouvert aux non-professionnels.

Pour répondre aux désirs des nombreuses adhérentes, la Société a très tôt été divisée en sous-groupes de recherches, les uns temporairement rassemblés autour d'un objectif commun comme celui de publier une nouvelle traduction du «Deuxième sexe » entre 1988 et $1997^{3}$, les autres attelés à des sujets aussi variés que le travail féminin, le droit européen des femmes, l'étude comparée des féminismes ou l'histoire des femmes. Le travail de traduction y tient nécessairement une grande place. Résolument transculturelle, la SFJEF ne se cantonne pas aux études françaises mais s'intéresse aux problèmes auxquels les femmes des deux pays sont confrontées.

\section{Le groupe « histoire des femmes des $\mathrm{XIX}$ et $\mathrm{XX}$ siècles ». Attentes et difficultés}

6 Ce sous-groupe s'est constitué en 1987 autour d'Isabelle ${ }^{4}$, professeure, à cette date, d'histoire de la France à l'université Sophia de Tokyo. Organisées de manière très informelle, la dizaine de femmes réunies dans ce bureau facilement accessible au centre de Tokyo désiraient principalement créer un lieu de rencontre. Leur motivation 
essentielle était alors de renouer avec la langue et la culture françaises qui avaient été leur choix universitaire une vingtaine d'années plus tôt. Ainsi, Shizuko ${ }^{5}$ est « entrée dans ce groupe dans le but d'approfondir (ses) connaissances sur la vie et la pensée des Françaises du XIX ${ }^{e}$ siècle ». La possibilité de se réunir pour " prendre la parole » entre femmes était certainement sous-jacente dans l'esprit de chacune, mais non exprimée ; comme était sous-entendu un lien privilégié, mais mal défini, entre femmes et culture française. Pour Midori ${ }^{6}:$ « La France et les femmes sont deux mots très associés ».

7 Au début, la barrière des langues, les lacunes réciproques dans la connaissance de leurs histoires nationales furent les difficultés inhérentes à ce groupe de non professionnelles de l'histoire. Deux écueils menaçaient : faire évoluer ce groupe centré sur l'histoire vers une sorte de cours universitaire avec ce que cela suppose de routine académique et d'intimidation vis-à-vis de la libre parole ; verser, au contraire, dans un cercle de dames réunies autour d'un thé. Vingt ans plus tard, il est possible de dire que ces dangers furent largement évités. Yuko ${ }^{7}$ souligne combien «l'attrait du groupe tient d'abord à l'atmosphère chaleureuse et libre, où chaque membre peut poser des questions même simplettes, à la différence des cercles académiques si froids à l'égard des non-initiés ", ajoutant que « cela n'empêche pas que ce groupe d'études soit sérieux et méthodique ».

\section{Profils des participantes du groupe « histoire des femmes des XIXe et XXe siècles »}

8 Décrire succinctement le profil des membres du groupe est nécessaire pour comprendre les raisons de sa réussite. L'homogénéité du groupe est assez grande. Les participantes sont nées et ont grandi dans un des moments les plus bouleversés et les plus décisifs de l'histoire du Japon. Les anciennes, nées avant la guerre, ont vécu la défaite dans leur enfance, celles qui sont nées pendant la guerre ont connu les difficultés de la reconstruction économique, tandis que les plus jeunes ont goûté aux premiers bienfaits de la société de consommation. Ce décalage générationnel entraîne des points de vue légèrement différents sur l'histoire des femmes et du féminisme.

9 Leurs origines sociales sont relativement privilégiées. En remontant dans leurs familles, on rencontre des bushi (samouraï), des commerçants en gros de la grande ville d'Osaka, et des paysans. La promotion de la génération des parents s'est faite à travers la fonction publique et par le salariat d'entreprise. Toutes ont eu la chance de faire de bonnes études. L'avènement de la démocratie en 1945 sous la pression de l'occupant américain, la seconde grande ouverture à l'Occident, et le démarrage d'une des plus formidables sociétés de consommation au monde constituent le fond socio-historique absolument neuf sur lequel vont se détacher leurs histoires singulières. Mariko ${ }^{8}$ est "entrée à l'école primaire tout de suite après la défaite du Japon ». "On nous y apprenait l'égalité des sexes ", se rappelle-t-elle.

10 La nouvelle constitution de 1946 fonde le mariage sur le consentement mutuel de deux individus et le Code civil révisé (1947) libère la femme des servitudes du système féodal du $i e^{9}$. «Passées du statut de sujet à celui de citoyennes, nous avions obtenu l'égalité des sexes et le droit de vote, pourtant la famille comme la société restaient partagées entre ancienne et nouvelle morale", note Atsuko ${ }^{10}$. Malgré ces limites, les participantes profitent du nouveau système éducatif mis en place après la défaite: coéducation, 
égalité des sexes dans l'accès aux études, contenus et méthodes pédagogiques libérales ; toutes estiment avoir été les heureuses bénéficiaires de cette révolution scolaire. Mais ces immenses progrès n'ont pas entraîné un changement fondamental de l'image des filles dans l'opinion qui continue à penser leur rôle en termes de mariage et de maternité. Si, en théorie, l'accès à l'université était désormais ouvert aux filles comme aux garçons, en réalité, il était contrecarré par les obligations du mariage. Les participantes ont toutes pu fréquenter l'université, privilège qu'elles attribuent au fait d'avoir été des citadines et au soutien exceptionnel de leur famille.

C'est plutôt au moment d'entrer dans la vie active que les participantes ont découvert les limites de la démocratie affichée et le monde hostile de l'entreprise : examens de recrutement réservés aux garçons, désintérêt de l'entourage pour leurs projets, étroitesse des débouchés, conditions de travail inégales. Et surtout, pressions pour hâter le mariage. Ainsi Yoshiko ${ }^{11}$, soumise à l'autorité paternelle, malgré le très bon niveau de ses études : «Mon père s'est opposé à ce qu'une fille de bonne famille prenne un métier. Je me mariai donc en 1965 ».

12 Toutes les autres participantes ont pu travailler - jusqu'au mariage tout au moins -, souvent dans l'enseignement, domaine le plus accessible et le plus propre à concilier mariage et carrière, telles Shizuko qui choisira le célibat ou Mariko. Grâce à l'appui de son père, Midori devient professeur de musicologie, mais Naoko ${ }^{12}$ qui commence à enseigner la musique dans un jardin d'enfants est vite victime d'une restructuration. Les jeunes femmes célibataires ont été licenciées en priorité. Les autres participantes ont été embauchées par des entreprises, non sans grandes difficultés. Atsuko, renonçant à poursuivre ses études, est entrée dans la section des relations publiques d'une compagnie de commerce. Mais, là encore, tout fut fait pour la décourager : bas salaire, obstacles à l'avancement, pressions sur la vie privée. Atsuko, malade, s'est réfugiée dans le mariage. Hisako ${ }^{13}$ décroche un bon travail dans une entreprise chimique de Mitsubishi mais se souvient que «l'essentiel de son travail consistait à traduire brevets et divers documents ». Elle découvre l'énorme différence entre filles et garçons diplômés de l'université : «J'avais le statut d'auxiliaire B, au-dessus des filles qui avaient le bac, mais en dessous des garçons qui n'avaient que le bac. Et les filles étaient condamnées à rester auxiliaires $\mathrm{B} !$ ».

Hisako lutte d'abord en entrant dans le syndicat-maison, où elle est la première employée sortie de l'université à jouer un rôle important. Hélas, le mur d'opposition masculine a raison de ses efforts. Découragée, elle abandonne et se marie. Mais sa conscience féministe en sort éveillée. Yuko se marie en 1976 avec un étudiant de la même université. Elle note une amélioration des conditions de travail en entreprise après 1970, mais, elle-même, décide de se consacrer à sa famille. Les enfants élevés, elle a repris ses études universitaires. Elle se considère comme une femme au foyer typique du Japon contemporain.

14 Au moment d'entrer dans le groupe, l'aspiration de la plupart des participantes n'était pas de lier leurs parcours singuliers au travail de réflexion collective qu'elles allaient entreprendre. C'est la dynamique du groupe sur l'histoire des femmes qui, par la suite, leur fit apparaître ou réapparaître ces injustices subies dans leur jeunesse, et suscita le désir de les comprendre. 


\section{Choisir le français, significations}

15 Le choix de la littérature française, contre l'avis des parents parfois, n'est pas sans importance. Il doit être replacé dans la « vague française » de l'après-guerre. Libérés du militarisme par la défaite, nombre d'intellectuels japonais désireux d'échapper au dilemme Japon /États-Unis, ont été attirés par l'Europe, un monde qui n'était pas lié directement à la guerre faite et vécue par les Japonais, un monde qui bénéficiait d'une image de culture et de raffinement. «Ce fut un engouement pour l'existentialisme de Sartre et de Beauvoir. Ainsi, moi-même, j'ai choisi le Deuxième sexe pour mon mémoire de licence ». (Yoshiko)

Les romans français déjà traduits depuis l'ère Meij ${ }^{14}$ ont connu un regain de popularité. Dès l'école primaire, Atsuko se rappelle avoir lu Le Comte de Monte-Cristo, Les Misérables; au lycée : Stendhal, Romain Rolland, Martin du Gard; à l'université : La Bruyère, La Rochefoucault. Shizuko a lu en traduction George Sand, Balzac, Alexandre Dumas, Stendhal, Flaubert, Victor Hugo, Maupassant, Daudet, Romain Rolland, Camus... L'engouement touchait d'autres aspects de la culture française : « À l'époque où j'étais étudiante, les adultes rêvaient de la France (encore difficilement accessible) à travers les romans, le cinéma, les chansons françaises. La vie de Sartre et de Beauvoir étonnait, attirait. » (Shizuko)

17 Ainsi, toutes disent avoir choisi le français par désir de liberté, de rêve, de distinction. La langue française résonnait de façon harmonieuse et la mode représentait le chic enviable des Parisiennes. Le choix du français représentait également une forme de résistance à l'anglais, pourtant plus avantageux pour celles qui auraient envisagé une carrière dans la traduction ou l'enseignement. Pour certaines, l'étude de la littérature française touchait à des préoccupations existentielles. "J'ai découvert Simone de Beauvoir. J'ai alors réalisé pour la première fois que les femmes étaient capables de revendiquer leur indépendance » écrit Mariko.

Le thème de la recherche de l'identité féminine revient chez d'autres participantes. Comment construire son identité de femme dans la société japonaise de l'aprèsguerre? Quel rôle y jouer? Certaines ont commencé à chercher des réponses dans la littérature, comme Atsuko, «très attirée par les personnages féminins de Stendhal » et qui a "aimé ces auteurs car ils (l') ont fait réfléchir sur les fondements mêmes des relations entre individu et société ».

Le voyage en France devait être la conclusion désirée de quatre années d'études, chargé de multiples significations : poursuivre des études, s'éloigner de la famille, éprouver ses capacités d'indépendance et voir de ses yeux cette culture qui les attirait. Yoshiko passe un an à Paris où elle obtient le diplôme de l'École supérieure de préparation et de perfectionnement des professeurs de français à l'étranger de la Sorbonne. Shizuko, après six années de travail, quelques économies et le désir de voir Paris avant ses trente ans, s'envole enfin pour la France. Elle est étonnée d'y rencontrer des femmes mariées, cadres poursuivant leur travail, alors qu'au Japon, les femmes encore jeunes cessaient leur activité salariée pour se marier. "Je plaignais les femmes au foyer japonaises et j'imaginais leurs soucis, car dans mon enfance j'avais connu des veuves de guerre qui élevaient seules leurs enfants ».

Naoko, décidée à ne pas être happée par la famille, part en France à ses propres frais en 1979. Hisako, déjà mariée, fait son premier voyage en France en 1973 : deux semaines à 
Paris pour assister aux plus grands défilés de haute couture de la saison. Dès cette époque, elle cherche à reprendre une vie professionnelle dans le domaine de la mode. Elle y réussira. Atsuko, qui aurait aimé prolonger ses études de littérature, n'obtint le droit de voyager qu'après une guerre d'usure avec sa mère. Elle reste un an en France, de 1969 à 1970, et garde de sa vie d'étudiante à Tours puis à Grenoble un souvenir ému : «Pour moi, le séjour en France a représenté une émancipation de l'esprit et un enrichissement des sensations ». Mariko arrive en France juste après les événements de 1968, pour deux mois. Un voyage "volé » au devoir familial. Elle visite ensuite, en un mois, quatorze capitales européennes, "Je pensais que si je ne le faisais pas à ce moment-là, l'occasion ne me serait peut-être plus jamais offerte ».

21 Le voyage en France ainsi conquis, malgré un grand nombre de difficultés financières et familiales qui ne sont plus concevables aujourd'hui, reste un souvenir marquant de leur jeunesse et a certainement contribué au désir de former un groupe d'échange d'idées sur la vie et l'histoire des Françaises.

\section{Histoire et histoires personnelles}

Dès nos premières réunions où Française et Japonaises s'observaient discrètement, bien des choses nous apparurent vite communes, et d'abord l'histoire de ce milieu du XX siècle, ses modes culturelles et artistiques, ses mouvements d'étudiants, ses idées nouvelles sur les femmes; nous nous étonnions d'avoir vécu des deux côtés de la planète une chaîne d'événements semblables. Cet étonnement, le soulagement et le plaisir qui s'en suivirent de part et d'autre, nous permirent de nous interroger plus franchement sur nos différences. Isabelle exposa son choc culturel lorsque, après être entrée par son mariage dans une famille japonaise, elle découvrit la force des pressions familiales et l'absence d'alternative offerte aux femmes. Pourtant, les participantes ne faisaient nullement figure de victimes et, bien qu'arrêtées ou freinées dans leur élan vers plus d'ambition, aucune d'entre elles ne semblait amère, déçue ou révoltée. Aucune ne s'était d'ailleurs laissée complètement enfermée dans l'univers familial. Retrouver l'estime de soi, selon la formule de Yoshiko, la trop vite mariée, en retrouvant une activité intellectuelle propre, hors du domaine étroit des préoccupations du travail et de la famille mais néanmoins liée à leur «vécu» de femmes, avait été leur souci permanent. Celles qui avaient dû suivre un mari se sont enrichies de ces voyages; toutes étaient et sont restées sensibles et ouvertes à l'actualité. Toutes avaient déjà su trouver une forme d'expression personnelle, études, écriture, activités artistiques, sociales ou féministes quand elles sont entrées dans le groupe.

\section{Le temps de la lecture des autobiographies de Françaises : une période d'accumulation}

Les débuts du travail commun ont été empiriques. Qu'allions-nous étudier ensemble et selon quelle méthode? Nous nous appuyions sur quelques certitudes : une curiosité mutuelle, un désir d'échange sincère, une méfiance face aux poncifs des relations franco-japonaises, la modestie des non-professionnels. Dans ces tâtonnements, nous répétions spontanément les commencements de l'histoire des femmes. Il nous fallait nous appuyer sur du matériau, des textes qui nous intéressent en tant que femmes déjà 
loties d'un vécu non négligeable. C'est ainsi que nous eûmes l'idée de commencer par la lecture des autobiographies de Françaises des $\mathrm{XIX}^{\mathrm{e}}$ et $\mathrm{XX}^{\mathrm{e}}$ siècles.

Cette lecture s'avéra facile pour des grandes lectrices de romans français, attrayante et stimulante dans tous les détails qu'elle apportait sur les mœurs et sur les institutions sociales, politiques et religieuses de la France. Nous retrouvions l'enthousiasme des historiennes-défricheuses des années 1970. Les Japonaises découvraient que ces Françaises, particulièrement ces bourgeoises dont elles n'avaient eu qu'une image romancée, avaient une histoire complexe entre pouvoirs et servitudes : «Ce que j'ai découvert, c'est la réalité de la vie des bourgeoises du siècle dernier, prise pour modèle de la Femme idéale. Ce modèle semblait jusqu'à une date récente si évident qu'il cachait une réalité bien plus contrastée et plus douloureuse ». (Mariko)

Découvrir l'épaisseur de la vie de ces femmes jusque-là "invisibles » ou "mythifiées » fut une révélation et le véritable déclencheur de l'activité du groupe. L'intensité de la curiosité ainsi éveillée déboucha sur une période d'identification. Chacune se mit à étudier une mémorialiste proche de sa sensibilité ou de son expérience de femme. Naoko choisit: «d'étudier les Mémoires d'une paysanne bretonne de la première moitié $\mathrm{du} \mathrm{XX}^{\mathrm{e}}$ siècle, Yvonne Rioux. Plus que la vie de femmes célèbres, la vie des femmes ordinaires m'a paru proche ».

Se sont développées ainsi des suites superposées de dialogues entre Françaises et Japonaises, entre femmes d'hier et d'aujourd'hui. Dialogues exigeants car nous avions de nombreuses lacunes à combler dans nos connaissances historiques, mais passionnants car bien qu'amateur en histoire, chacune avait par ailleurs une bonne formation universitaire dans des domaines aussi variés que la littérature, la musique, la mode, le droit international : « Dans nos séances d'études, les membres partagent leurs connaissances et leurs points de vue en les faisant converger sur le sujet commun choisi ensemble ", écrit Yuko, exprimant parfaitement l'originalité de notre tentative entre recherche et vécu :

Ce qui me semble caractériser le groupe, c'est le fait que tous les membres sont des femmes qui ont une vie pleine et ancrée dans la société contemporaine, qu'elles ont crée et élevé une famille, ont travaillé, sont des personnes qui ont des idées bien enracinées dans la réalité, fondées sur leurs expériences heureuses et malheureuses. Cela me semble important car on ne peut faire l'histoire des femmes sans en connaître la vie la plus ordinaire. À la différence de l'histoire des hommes pour laquelle on peut toujours se rabattre sur la vie publique.

À partir du moment où chacune a eu une connaissance assez approfondie d'une mémorialiste et après avoir mis en commun ces découvertes, le sens de notre activité s'est précisé. Ce travail nous passionnait parce qu'Histoire et histoires singulières se croisaient, s'éclairaient réciproquement, comme s'éclairaient mutuellement l'histoire des Françaises et celle des Japonaises. Pour achever ce temps d'accumulation et d'identification, nous avons voulu faire le point dans un article intitulé «Portraits de femmes par elles-mêmes " paru dans Espace des femmes en 1997. Tout au long de ce travail commun, l'atmosphère de notre groupe s'est modifiée, de plus en plus libre, chaleureuse et festive. Même les plus réservées d'entre nous ont souvent pris la parole : « Discuter en groupe et prendre la parole devant les autres pour exprimer son point de vue. J'ai pu ainsi élargir ma pensée sur de nombreux problèmes concernant les femmes et le féminisme. C'est très réjouissant. » (Yoshiko). Une solidarité féminine se forgeait : "J'aime la cordialité de nos réunions où chacune peut poser des questions naïves et parler comme elle veut. Ces questions naïves sont en fait liées à de grands problèmes, 
plus larges. Chaque membre apporte non seulement ce qu'elle est mais un peu de ce qu'était sa mère, ou sa grand-mère ». (Mariko)

\section{Le temps des questionnements : de la difficulté des comparaisons}

Tout naturellement, l'intérêt avivé par les textes français entraîna le désir d'explorer le côté japonais : «J'ai commencé à découvrir l'histoire de la vie privée et de l'éducation des Françaises, parallèlement, j'ai commencé à m'intéresser à l'histoire des Japonaises et j'ai eu envie d'en savoir plus sur leur vie réelle et concrète" (Atsuko). Les autobiographies étudiées avaient mis en évidence les efforts et les difficultés des Françaises pour accéder à la modernité, et faisaient écho aux profonds bouleversements sociaux et familiaux de l'histoire des Japonaises: «J'ai commencé par l'étude de la vie des Françaises et j'en suis à présent à réfléchir sur ma propre famille et sur les bouleversements qu'elle a traversé depuis la guerre ». (Naoko)

Pour ce premier travail de comparaison, nous avons choisi l'éducation des filles, sujet très présent dans les autobiographies, mais sujet délicat puisque l'éducation reçue est l'armature même de notre conscience nationale, point aveugle de nombre de recherches. Ce travail, ardu mais excitant, donna au groupe un second souffle. Mariko découvre "la difficulté et le plaisir de faire une recherche collective » et le goût de l'histoire : "Quand nous avons essayé de retracer l'histoire de l'éducation des Japonaises depuis Meiji, la chronologie qui m'avait d'abord paru aride, s'est animée et a commencé à devenir une histoire vivante pour moi grâce à nos discussions et au partage des tâches au sein du groupe ».

Le travail du groupe s'affina. Les Japonaises se réapproprièrent une histoire des femmes très peu enseignée et celle de leur famille, dont elles publièrent une partie des résultats en 2003 dans Espace des femmes sous le titre «Bonne épouse, mère avisée, un destin contesté au Japon et en France». Ensemble, nous avons vu la similitude du développement de ces éducations nationales liées aux causes patriotiques et économiques, et les dissemblances importantes dues au substrat culturel et familial: Ainsi remarque Atsuko, "George Sand et Nogami Yaeko ${ }^{15}$ bien qu'ayant vécu à des périodes et dans des cultures différentes, ont à mes yeux une personnalité semblable, mais ce qui les différencie, c'est la question religieuse. Quand je lis les autobiographies de Françaises, ce qui m'étonne le plus, c'est l'éducation religieuse reçue dans la jeunesse, leur crise de mysticisme au couvent ».

31 Ce travail de comparaison, toujours en cours, nous a fait franchir une nouvelle étape dans l'histoire des femmes en associant toute recherche sur les femmes à la connaissance globale du fonctionnement sexué de nos sociétés: "Sur le fond, j'ai commencé à comprendre combien les institutions pouvaient contraindre les citoyens et combien l'éducation est l'enjeu de conflits violents » (Mariko). Décortiquer les systèmes d'oppression à partir de nos expériences vécues, mais en les situant dans l'histoire comparée des deux pays est maintenant notre objectif.

32 Voici l'histoire d'un groupe d'études amateur qui s'était constitué dans le simple but de développer des échanges culturels et amicaux, sans projet explicitement féministe au départ et qui se retrouve aujourd'hui, après un long parcours, principalement intéressé par la question des femmes dans l'histoire de nos deux sociétés. Réappropriation, 
débats et nouveaux questionnements, le groupe a fait siennes les étapes franchies par l'histoire des femmes. Chaque femme du groupe s'est racontée, révélant ou omettant avec liberté des pans de sa vie, avançant d'abord masquée derrière le texte autobiographique d'une autre, puis à visage découvert lorsqu'elle a eu l'assurance d'être comprise et le sentiment de pouvoir, par sa prise de parole, devenir actrice de l'histoire des femmes, donc de l'Histoire : «J'ai appris à mieux me connaitre... Je suis heureuse de pouvoir étudier avec des femmes qui ont une histoire personnelle différente de la mienne, dans une ambiance franche et libre» (Atsuko). Méthode proche de l'histoire orale, avec sa saveur unique, libératrice, mais aussi ses dangers de débordements subjectifs. Dangers qui ont été pour une grande part évités par la confrontation permanente des points de vue - tout le monde est à la fois questionneur et questionné - et par les recherches objectives communes qui ont pu aboutir à des publications communicables à d'autres femmes.

\section{BIBLIOGRAPHIE}

«Espaces des Femmes » : bulletin annuel de la SFJEF. Les numéros 10 (1993) et 20 (2003) donnent des résumés conséquents des objectifs, fonctionnement, liste des membres et sujets traités par la Société.

Actes des recherches collectives franco-japonaises : « Pour la comparaison franco-japonaise des études sur les femmes : sur la nécessité des nouvelles méthodes comparatives » in Société Francojaponaise des Études sur les Femmes, édité par les soins de La Maison Franco-japonaise de Tokyo, 2002.

Des Japonaises, collectif, Paris, Des femmes, 1987.

Japanese women, New feminist perspectives on the past, present and future, edited by Kumiko FujiwaraFanselow and Atsuko Kaneda, in The Feminist press at the City University of New York 1995.

SATO Barbara, The New Japanese Woman. Modernity, media, and women in interwar Japan, Duke University Press, London, 2003.

THÉBAUD Françoise, Écrire l'histoire des femmes, Paris, ENS éditions, 2001.

UENO Chizuko, The Japanese Women's movement : The counter-Values and industrialism in the Japanese trajectory: Modernization and beyond. New York, Cambridge University press, 1988.

\section{NOTES}

1. L'idée première de cet article est née du désir du groupe de répondre au questionnement de la sociologue française Anne Marie Devreux intéressée par cette démarche collective francojaponaise sur l'histoire des femmes. Chaque membre a écrit en toute liberté à propos des raisons variées qui avaient motivé sa participation et les bienfaits qu'elle estimait avoir recueillis. Ces témoignages autobiographiques ont ensuite été mis en commun, analysés et longuement discutés 
par l'ensemble des participantes. La traduction et la coordination définitive ont été assurées par Isabelle Hasegawa en 2004.

2. 3 Société Franco-japonaise des Études sur les Femmes. Siège : Maison Franco-japonaise 3-9-5 Ebisu, Shibuya-ku, 152-0062 Tokyo ; Site: http://cdfjf-fr.blogspot.com/

3. Le Deuxième Sexe (1949) fut traduit en japonais en 1953, la même année qu'aux États-Unis.

4. Hasegawa Isabelle, diplômée en histoire, professeure à l'université Sophia de Tokyo, mariée, un enfant, fait des recherches sur l'histoire des femmes des XIX ${ }^{\mathrm{e}}$ et $\mathrm{XX}^{\mathrm{e}}$ siècles.

5. Onuma Shizuko, diplômée en littérature française, célibataire, vit à Tokyo enseigne le français à l'université de Hosei de Tokyo. Shizuko s'intéresse à toutes les expressions de la culture française, en particulier, le cinéma.

6. Kobayashi Midori, diplômée en musicologie, mariée, sans enfant, habite à Tokyo, enseigne la musicologie à l'université de musique de Kunitachi, poursuit ses recherches sur les compositrices méconnues et organise des concerts dans le monde entier pour les faire connaître.

7. Matsuda Yuko, diplômée en études des relations internationales, mariée, deux enfants, habite à Osaka, continue ses recherches sur la vie des bourgeoises françaises au XIX ${ }^{\mathrm{e}}$ siècle. Enseigne l'histoire à l'université féminine Shoin de Osaka.

8. Sano Mariko, diplômée en littérature française, enseigne le français à l'université Keio de Tokyo, mariée, sans enfant, habite à Kamakura, poursuit ses recherches sur les écrivaines françaises et étrangères actives à Paris dans la seconde moitié du XIX ${ }^{\mathrm{e}}$ siècle. Elle a écrit plusieurs articles sur Marthe Bibesco.

9. Ie désigne la «maison ». Dans l'ancien droit civil japonais, le père, chef de la famille de la «maison», jouit d'une autorité absolue sur tous ses membres. La femme et les enfants (les garçons jusqu'à 30 ans et les filles jusqu'à 25 ans) ne possèdent aucune autonomie. En 1947, le code Meiji (1893/1898) fut radicalement modifié, proclamant l'égalité de l'homme et de la femme au sein du couple, supprimant les contraintes de la ie et ouvrant ainsi la voie à la famille nucléaire moderne.

10. Nakamori Atsuko, diplômée en littérature française, mariée, un enfant, habite Tokyo. Elle a travaillé en entreprise, enseigne l'art de la broderie scandinave, un domaine qui est lié au Japon à la recherche sur les cultures féminines traditionnelles.

11. Matsuoka Yoshiko, diplômée en littérature française, mariée, deux enfants, a traduit des ouvrages américains sur l'autisme et continue de s'intéresser à ce domaine.

12. Kato Naoko, diplômée en pédagogie musicale, a enseigné la musique puis a travaillé dans une entreprise française au Japon. Célibataire, elle habite à Nagoya, souhaite étudier les questions relatives à la famille.

13. Hiki Hisako, diplômée en histoire des idées sociales françaises, a travaillé en entreprise. Mariée, deux enfants, elle habite Tokyo où elle enseigne la culture du vêtement.

14. L'ère Meiji, du nom de l'empereur Meiji, 1868 à 1912, marque la réouverture du Japon à l'Occident.

15. Célèbre écrivaine japonaise 1885-1995.

\section{RÉSUMÉS}

En 1983, un groupe de femmes japonaises francophiles, très intéressées par le féminisme français et les changements rapides de la vie des femmes en France depuis les années 1970, créent la 
Société Franco-japonaise des Études sur les Femmes. Le but est de promouvoir une meilleure compréhension entre les deux cultures et de développer des liens avec les Françaises sur un grand nombre de questions féminines. Le projet se révéla vite être un succès. Parmi les études entreprises par la Société, l'analyse comparée de l'histoire des Françaises et des Japonaises, domaine très peu exploré alors, a suscité un vif intérêt chez les participantes. Cet article revient sur vingt années de réflexion, de recherche, et d'échange par un groupe d'historiennes nonprofessionnelles sur les relations entre histoire et expériences de vie personnelles. Il nous montre comment l'étude de l'histoire des femmes dans un contexte international peut être fructueuse et bénéfique, et combien une libre discussion collective, sans tabous, sur ce sujet peut éveiller ou approfondir la conscience féministe.

In 1983, a group of francophile Japanese women, very interested in French feminism and in the rapid changes of French society since the 1970s, established the Franco-Japanese Society for Women's Studies. The group sought to promote a better understanding between the two cultures and to develop ties with French women on a number of feminine issues. The project encountered rapid success. Among the studies undertaken by the group was a comparative analysis of French and Japanese women's history, a little explored field whose results generated considerable interest among the members. This article analyzes twenty years of reflection, research and exchange by a group of non-professional historians about the interconnections between history and personal life experiences. It reveals how studying women's history in an international context can be fruitful and rewarding, and how the open discussion of these matters together can create or deepen feminist consciousness.

\section{INDEX}

Index géographique : Japon

Mots-clés : comparatisme, histoire des femmes, Japonaises, autobiographie

\section{AUTEUR}

\section{GROUPE HISTOIRE DES FEMMES}

Hasegawa Isabelle, Hiki Hisako, Kato Naoko, Kobayashi Midori, Matsuda Yuko, Matsuoka Yoshiko, Nakamori Atsuko, Onuma Shizuko, Sano Mariko. Le groupe « Histoire et Femmes », membre de la Société Franco-japonaise des Études sur les Femmes (SFJEF) dont le siège est situé à la Maison Franco-japonaise de Tokyo, a été fondé en 1983 dans le but de mener des études comparatives sur l'histoire des femmes des deux pays. Depuis plus d'une vingtaine d'années, les participantes, universitaires, femmes au foyer, étudiantes, dont le nombre oscille autour d'une quinzaine de membres, poursuit un travail de lecture, d'analyse comparée, et de traduction d'autobiographies de femmes françaises et japonaises. Les résultats de ce travail collectif ont été régulièrement publiés dans la revue de la Société "Espace des femmes". Isabelle Hasegawa anime ce groupe depuis ses débuts comme coordinatrice et traductrice. 